\title{
Embedding of Poly Honeycomb Networks and the Metric dimension of Star of David Network
}

\author{
${ }^{1}$ F.Simonraj and ${ }^{2}$ A.George \\ ${ }^{1}$ Department of Mathematics, Hindustan University, Chennai, India. \\ simonr@hindustanuniv.ac.in \\ ${ }^{2}$ Department of Information Technology, Hindustan University, Chennai, India. \\ ageorgeahindustanuniv.ac.in
}

\begin{abstract}
In this paper, we have introduced few Interconnection Networks, called David Derived Network $D D(n)$, Dominating David Derived Network DDD(n), Honeycomb cup Network HCC $(n)$ and Kite Regular Trianguline Mesh $\operatorname{KRrTM}(n)$. We have given drawing algorithm for $D D D(n)$ from Honeycomb network $H C(n)$ and embedded poly-Honeycomb Networks, $\operatorname{KRTM}(n)$ in to Dominating David Derived Networks. Also we have investigated the metric dimension of Star of David network $S D(n)$ and lower bound of the metric dimension for $\mathrm{DD}(n)$.
\end{abstract}

\section{Keywords}

Dominating David Derived Networks, Embedding, Honeycomb Networks, Kite Regular Triangulene Mesh, Metric dimension, Poly Honeycomb Mesh, Star of David network.

\section{INTRODUCTION}

In interconnection networks, the simulation of one architecture by another is important. The problem of simulating one network by another is modeled as a graph embedding problem. We know that the communication pattern of an algorithm can be modeled by a graph. Thus, the implementation of an algorithm in a system is an embedding of communication pattern of the algorithm into the network. There are several applications that can be modeled as a graph embedding problem. For example, the problem of finding efficient storage representations for data structures, where both storage representations and data structures are represented as graphs, is also reduced to a graph embedding problem. The problem of laying out circuits on VLSI chips can also be formulated as a graph embedding problem.[1].

A metric basis for a graph $G$ is a subset of vertices $W \subseteq V$ such that for each pair of vertices $u$ and $v$ of $V \backslash W$, there is a vertex $w \in W$ such that the distance between $u$ and $w$ is not equal to the distance between $v$ and $w$ that is $d(u, w) \neq d(v, w)$. The cardinality of a metric basis of $G$ is called metric dimension and is denoted by $\beta(G)$. The members of a metric basis are called landmarks. A metric dimension problem is to find a metric basis.

\subsection{OVERVIEW}

Graph embeddings have been well studied for meshes into crossed cubes [16], binary trees into paths [12], binary trees into hypercubes [11,15], complete binary trees into hypercubes[17], incomplete hypercube in books [13], tori and grids into twisted cubes [18], meshes into locally

DOI : 10.5121/jgraphoc.2012.4402 
International journal on applications of graph theory in wireless ad hoc networks and sensor networks (GRAPH-HOC) Vol.4, No.4, December 2012

twisted cubes [19], meshes into faulty crossed cubes [20], generalized ladders into hypercubes [21], grids into grids [22], binary trees into grids [23], hypercubes into cycles [24,25], star graph into path [26], snarks into torus [27], generalized wheels into arbitrary trees [28], hypercubes into grids [6], $m$-sequential $k$-ary trees into hypercubes [29], meshes into Möbius cubes [30], ternary tree into hypercube [31], enhanced and augmented hypercube into complete binary tree [32], circulant into arbitrary trees, cycles, certain multicyclic graphs and ladders [33], hypercubes into cylinders, snakes and caterpillars [34]. In this paper, we give some embeddings of Poly Honeycomb networks into $D D D(n)$ and $D D D(n)$ into Dominating Silicate network $D S L(n)$ for particular dimensions.

The first paper on the notion of metric basis appeared as early as 1975 under the name 'locating set' $[52]$. Slater [52,53] introduced this idea to determine uniquely the location of an intruder in a network[55]. Harary and Melter [46] and Khuller et al. [48] discovered this concept independently and used the term metric basis. This concept was rediscovered by Chartrand et al. [54] and also by Johnson [47] of the Pharmacia Company while attempting to develop a capability of large datasets of chemical graphs. It was noted in [45] that determining the metric dimension problem (resolving number) of a graph is an $N P$-complete problem. It has been proved that this problem is $N P$-hard [48] for general graphs. Manuel et al. [49] have shown that the problem remains $N P$-complete for bipartite graphs. This problem has been studied for trees, multi-dimensional grids [48], Petersen graphs [42], torus networks [51], Benes networks [49], honeycomb networks [50], enhanced hyper cubes [43], and Illiac networks [44]. In this paper we have investigated the metric dimension of $S D(n)$ and lower bound for the metric dimension of $D D(n)$.

\section{Definition: 1.1}

Let $G$ and $H$ be two finite graphs with $\gamma$ vertices. $V(G)$ and $V(H)$ denote the vertex set of $G$ and $H$ respectively. $E(G)$ and $E(H)$ denote the edge set of $G$ and $H$ respectively. An embedding from $G$ to $H$ is defined [2] as follows.

1. $f$ is a bijective map from $V(G) \rightarrow V(H)$

2. $f$ is a one to one map from $E(G)$ to $\left\{P_{f}(f(u), f(v)): P_{f}(f(u), f(v))\right.$ is a path in $H$ between $f(u)$ and $f(v)\}$.

Dilation of embedding of $G$ in to $H$ is given by $\operatorname{Dil}(f)=\operatorname{Max}\left\{\left|P_{f}(f(u), f(v))\right|:(u, v) \in E(G)\right\}$. Where $\left|P_{f}(f(u), f(v))\right|$ denotes length of the path $P_{f} \quad$ in $H$. Then the dilation of $G$ in to $H$ is defined as $\operatorname{Dil}(G, H)=\min \operatorname{Dil}(f)$. Where the minimum is taken over all embedding $f$ of $\mathrm{G}$ in to H. Embedding $G$ into $H$ with minimum dilation is important for network design and for the simulation of one computer architecture by another [3]. Embeddings as mathematical models of parallel computing have been discussed extensively in the literature [4,5]. In these models, both the algorithm to be implemented and the interconnection network of the parallel computing system are represented by graphs. The implementation details are then studied through the embedding.

\subsection{STAR OF DAVID}

In this section we consider Star of David which is a hexagram [8]. 
International journal on applications of graph theory in wireless ad hoc networks and sensor networks (GRAPH-HOC) Vol.4, No.4, December 2012

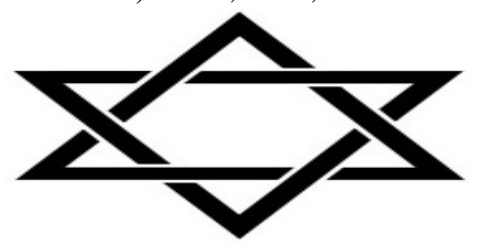

Figure1. Star of David.

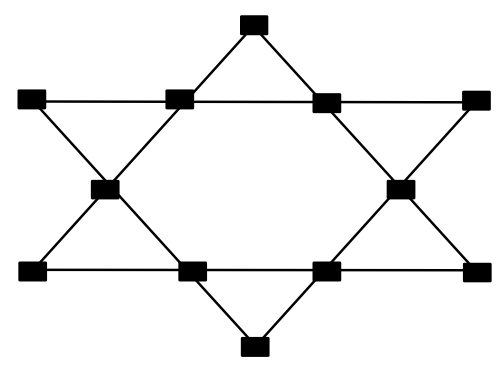

[6] Figure 2. Star of David graph

Here after we call this graph as Star of David network with dimension one $S D(1)$.

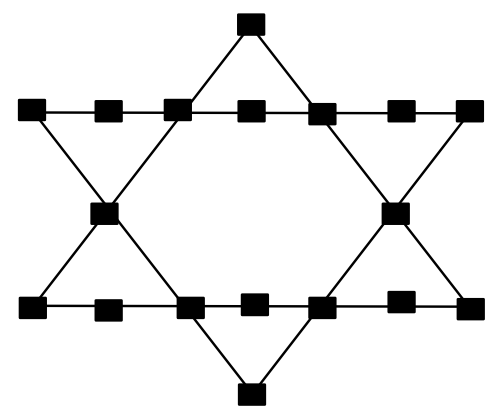

Figure3. David Derived graph (or Network) of dimension one. $D D(1)$

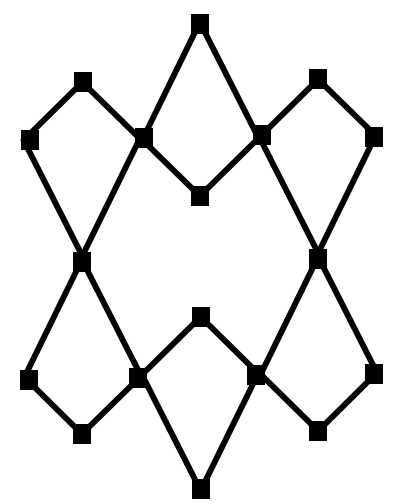

Figure 4. Isomorphic graph of $D D(1)$.

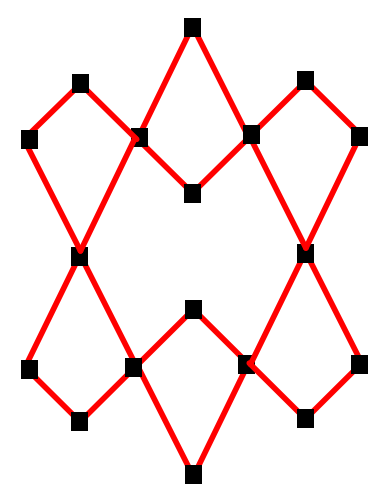

Figure 5. David Derived network $D D(1)$ 
International journal on applications of graph theory in wireless ad hoc networks and sensor networks (GRAPH-HOC) Vol.4, No.4, December 2012

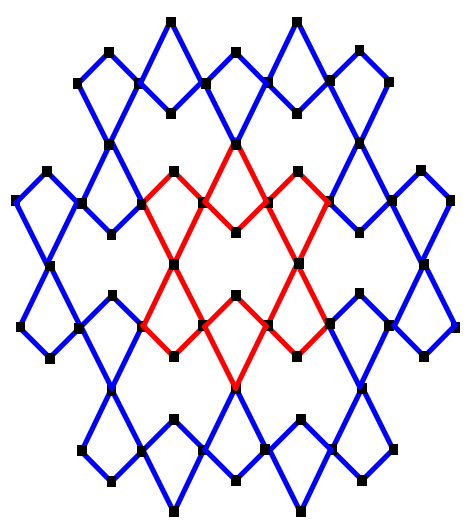

Figure 6. David Derived network of dimension two $D D(2)$.

\section{DRAWING ALGORITHM FOR $D D D(n)$}

Step-1: Consider a honeycomb network $H C(n)$ of dimension $n$.

Step-2: Split each edge of $H C(n)$ into two by inserting a new vertex .

Step-3: In each hexagon cell, connect the new vertices by an edge if they are at a distance of 4 units within the cell.

Step-4: Place vertices at new edge crossings.

Step- 5: Remove initial vertices and edges of Honeycomb network.

Step- 6: Split each horizontal edge into two edges by inserting a new vertex. The resulting graph is called Dominating David Derived network.

\subsection{Drawing method of $D D D(2)$ from $H C(2)$}

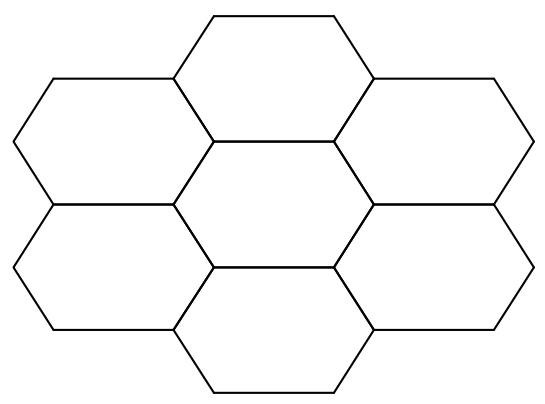

Figure 7. Step-1

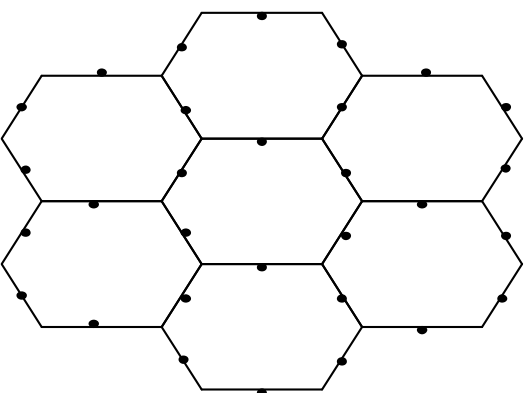

Figure 8. Step-2 
International journal on applications of graph theory in wireless ad hoc networks and sensor networks (GRAPH-HOC) Vol.4, No.4, December 2012

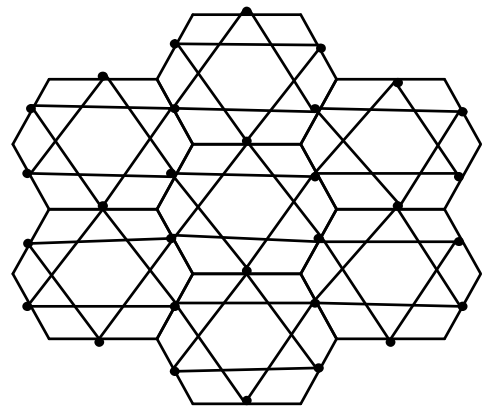

Figure 9. Step-3

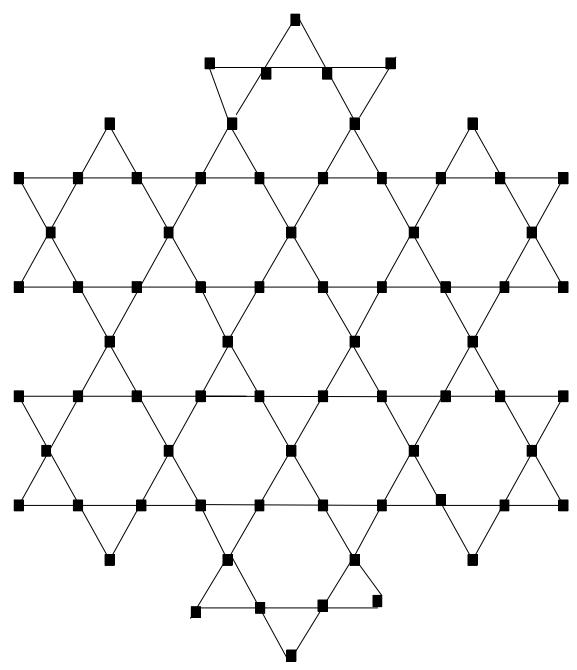

Figure 11. Step-5

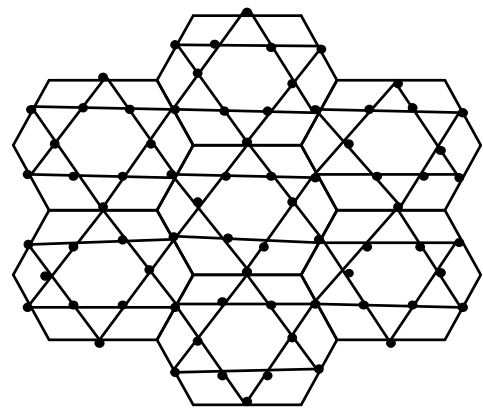

Figure 10. Step-4

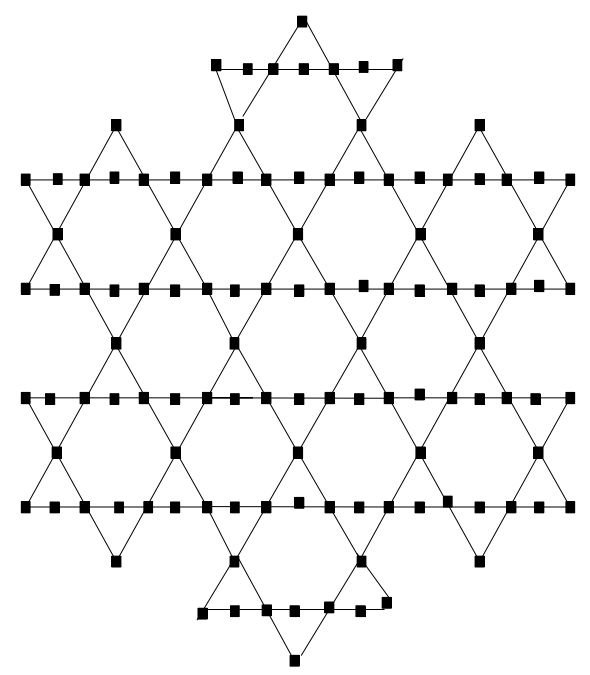

Step-6

Figure 12. $D D D(2)$

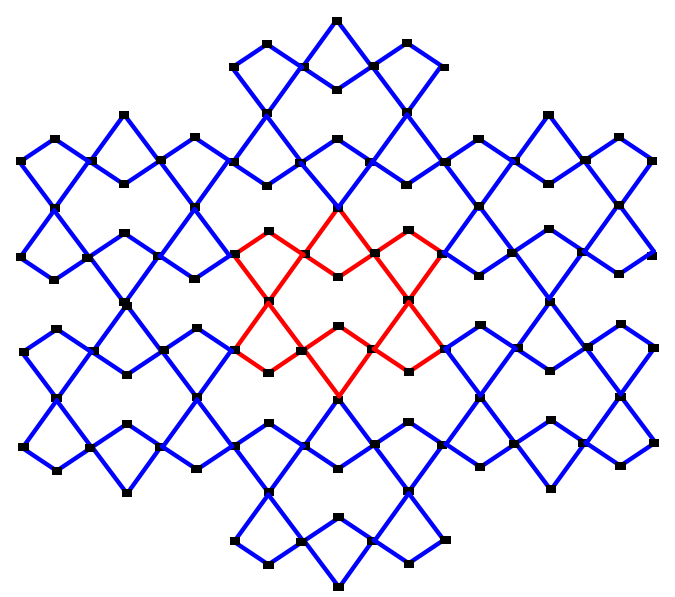

Figure 13. Isomorphic graph of $D D D(2)$ 
International journal on applications of graph theory in wireless ad hoc networks and sensor networks

(GRAPH-HOC) Vol.4, No.4, December 2012

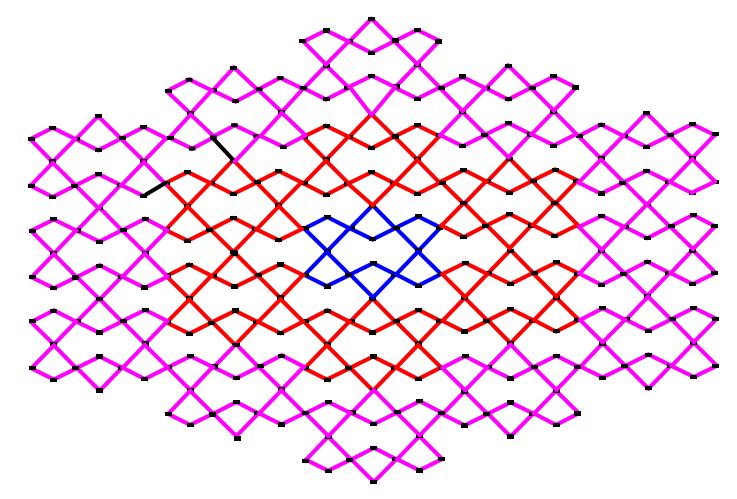

Figure $14 D D D(3)$

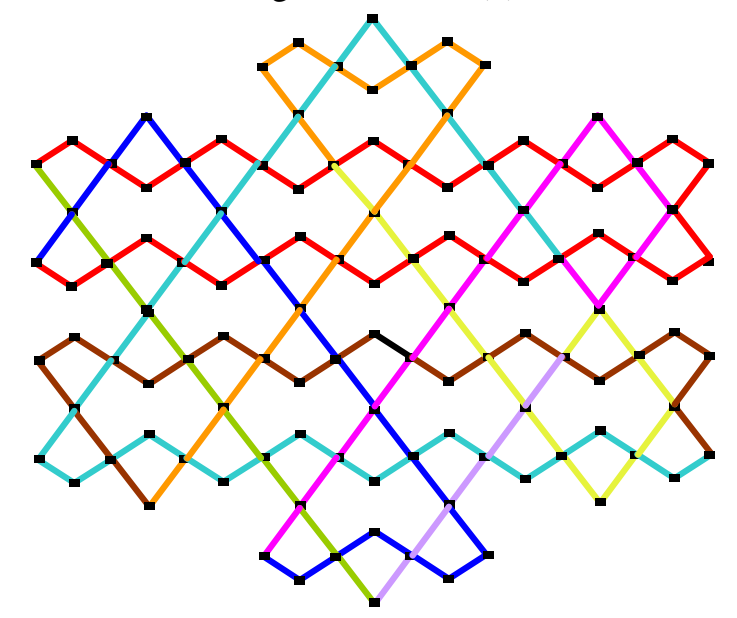

Figure15. Euler circuit of $D D D(2)$

Blue $\rightarrow$ Pink $\rightarrow$ Aqua $\rightarrow$ Brown $\rightarrow$ Orange

$\rightarrow$ Yellow $\rightarrow$ lavender $\rightarrow$ Lime $\rightarrow$ Red $\rightarrow$ Blue.

The first Dominating David Derived network $D_{1}(1)$ can be obtained by connecting vertices of degree two by an edge, which are not in the boundary or in unbounded dual of $\mathrm{DD}(1)$. See figure16.

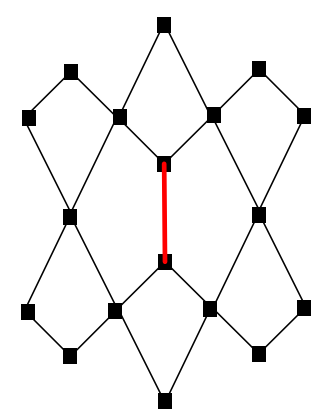

Figure16. $D_{1}(1)$ 
International journal on applications of graph theory in wireless ad hoc networks and sensor networks (GRAPH-HOC) Vol.4, No.4, December 2012

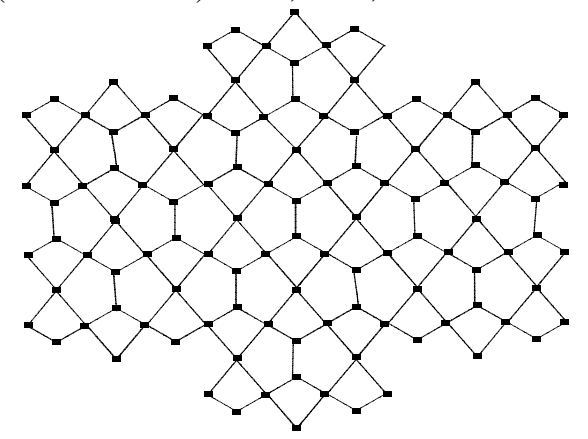

Figure 17. $D_{1}(2)$

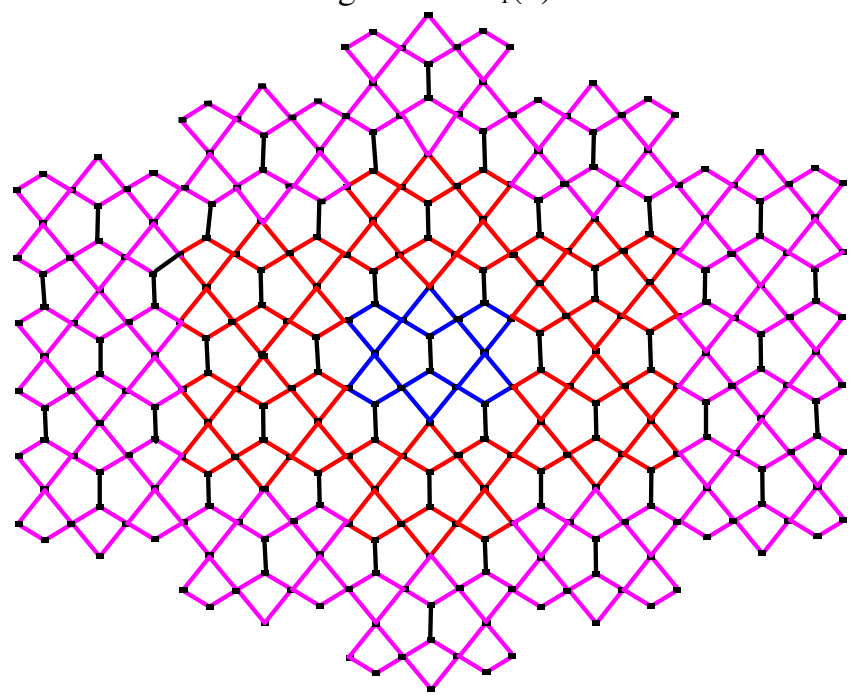

Figure 18. $D_{1}(3)$

Second Dominating David derived network of dimension one $D_{2}(1)$ can obtained by sub dividing once the new edge introduced in $D_{1}(1)$. See Figure 19. Third Dominating David derived network of dimension one can be obtained from $D_{1}(1)$ by introducing parallel path of length 2 between vertices of degree two which are not in boundary. See figure 20 (b) for third Dominating David Derived network of dimension two $D_{3}(2)$.

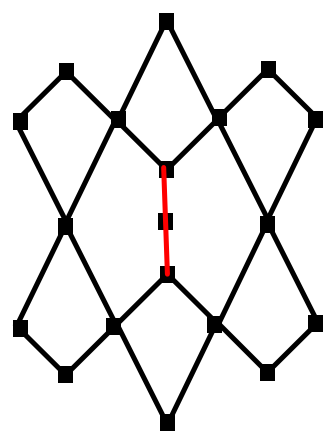

Figure 19. $D_{2}(1)$ 
International journal on applications of graph theory in wireless ad hoc networks and sensor networks (GRAPH-HOC) Vol.4, No.4, December 2012

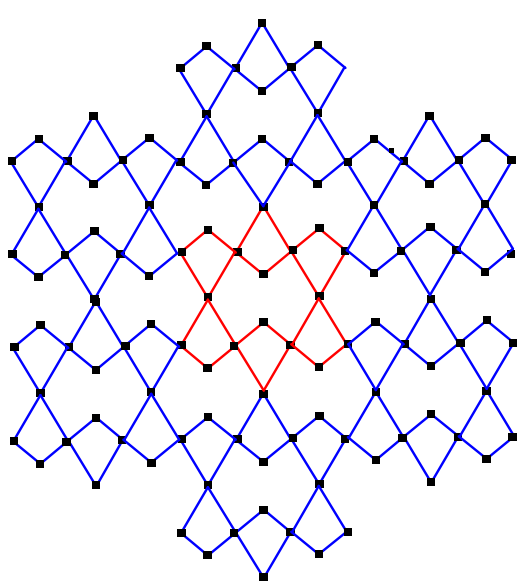

Figure 20(a) $D D D(2)$

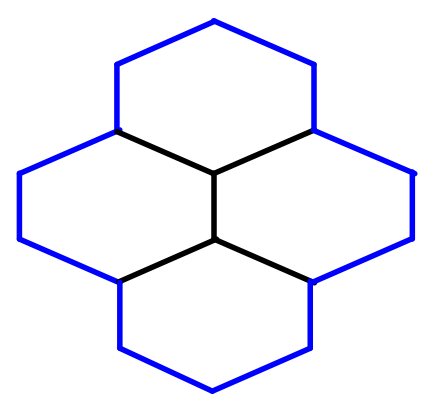

Figure 21(a) Honeycomb cup network $H C C(1)$

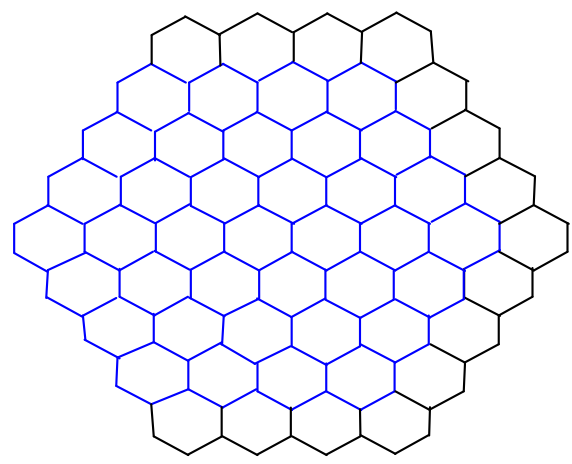

Figure 22(a). $H C(4)$ as a sub graph in $H C C(4)$

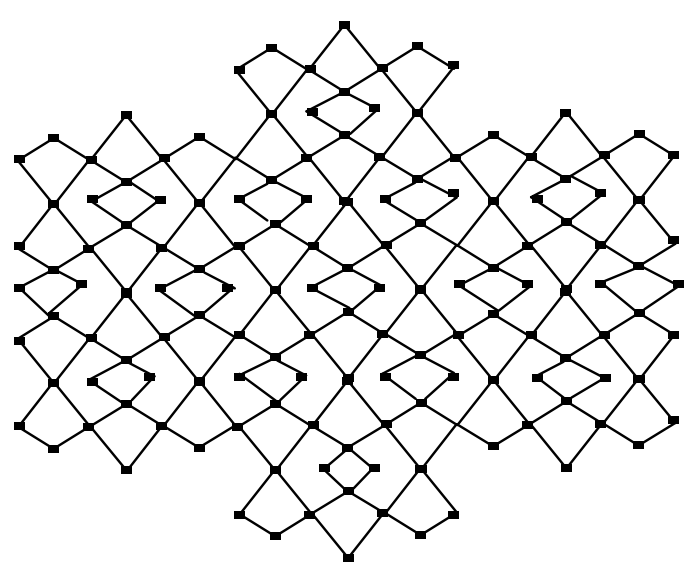

Figure 20(b) $D_{3}(2)$

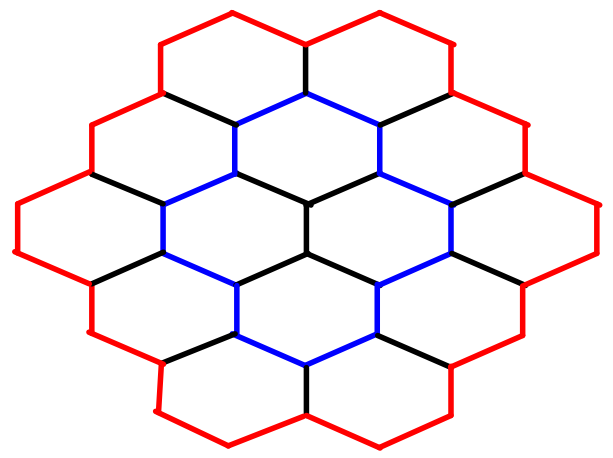

Figure 21 (b) $H C C(2)$

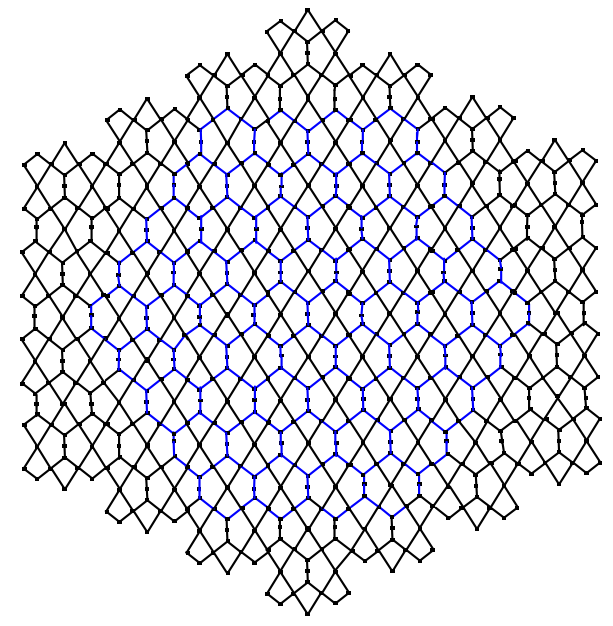

(b) $H C C(4)$ embedded in $D_{2}(4)$ 
International journal on applications of graph theory in wireless ad hoc networks and sensor networks (GRAPH-HOC) Vol.4, No.4, December 2012
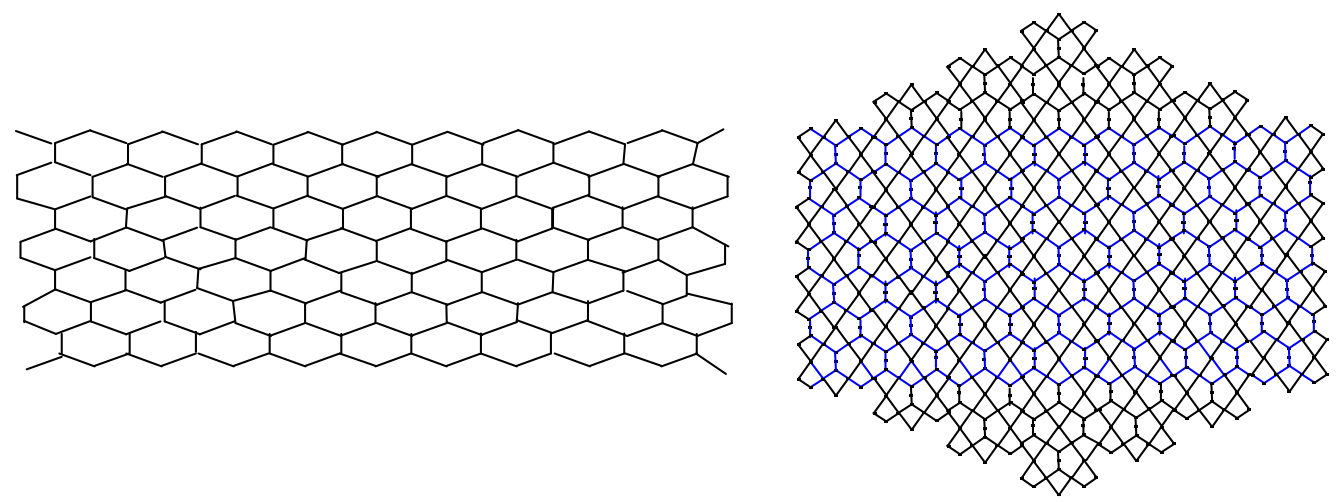

Figure23. HReM $(11,8)$ is embedded in $D_{2}(4)$ with dilation 2.
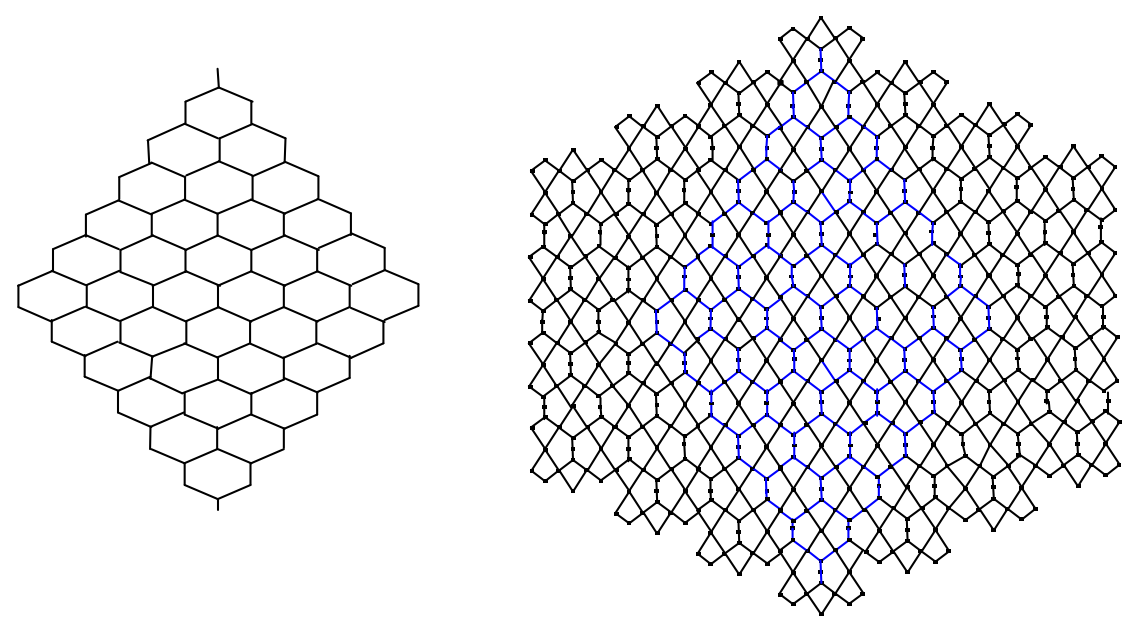

Figure 24.HRoMs(7) is embedded in $D_{2}(4)$ with dilation 2.

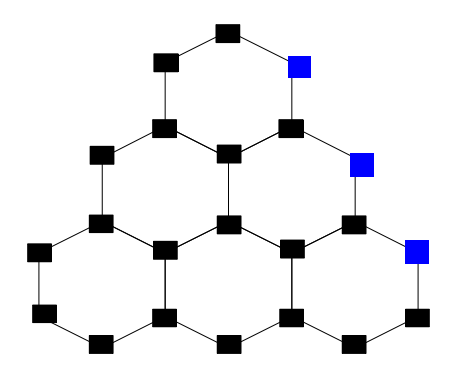

Figure 25. Honeycomb Regular Triangulene Mesh HRrTM(3). 
International journal on applications of graph theory in wireless ad hoc networks and sensor networks (GRAPH-HOC) Vol.4, No.4, December 2012

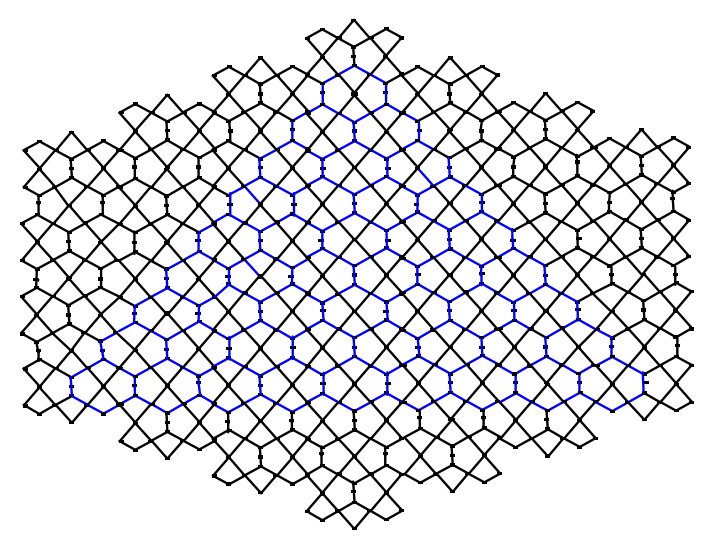

Figure26. $\operatorname{HR} T M(9)$ embedded in $D_{2}(4)$ with dilation 2.

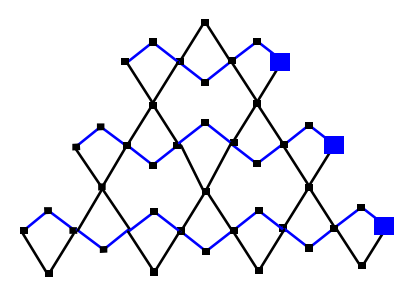

Figure27. Kite Regular Trianguline Mesh $\operatorname{KRrTM(3)}$

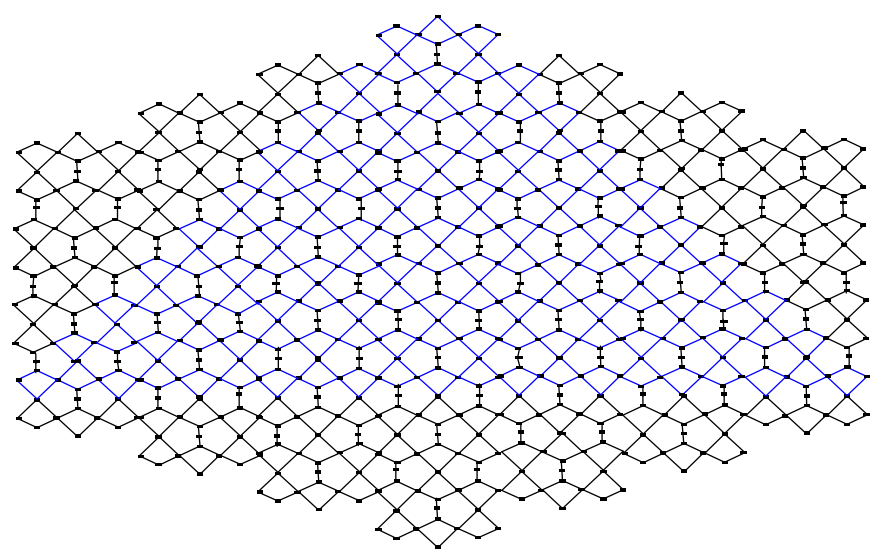

Figure 28. $\operatorname{KRrTM}(10)$ is embedded in $D_{2}(4)$ with dilation 2. 
International journal on applications of graph theory in wireless ad hoc networks and sensor networks (GRAPH-HOC) Vol.4, No.4, December 2012
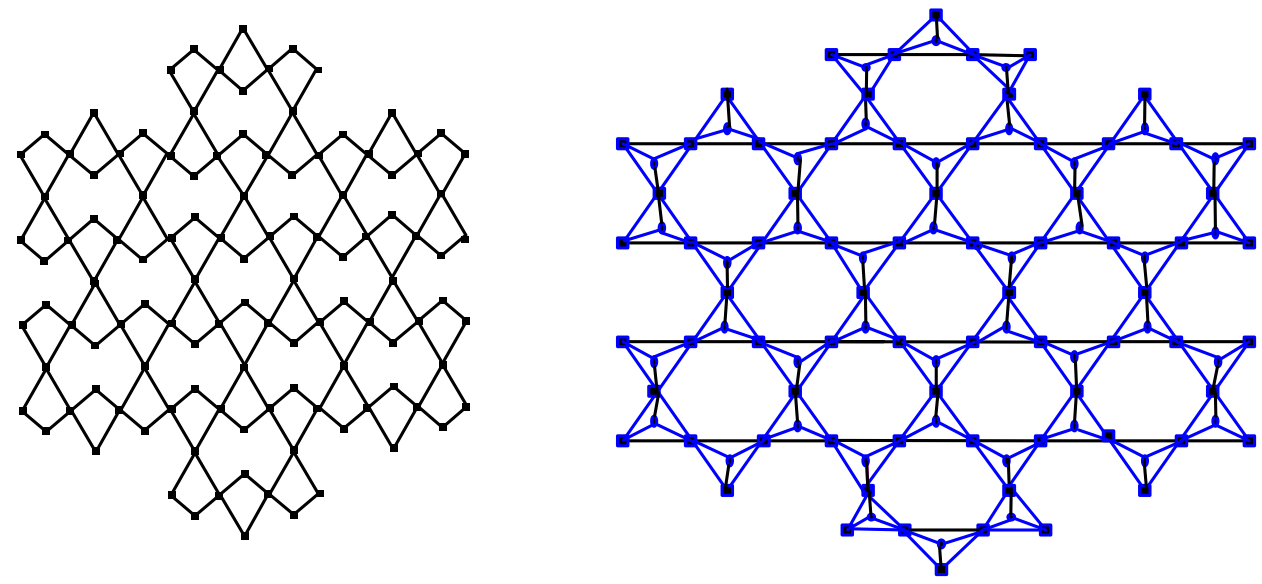

Figure 29. $D D D(2)$ is embedded in to Dominating Silicate Network $D S L(2)$ with dilation 1.

\section{COMPARISON OF NETWORKS}

TABLE 1

\begin{tabular}{|c|c|c|c|}
\hline Network & Vertices & Edges & Faces \\
\hline$[7] H R e M\left(t^{\prime}, t^{\prime \prime}\right)$ & $2 t^{\prime} t^{\prime}$ & $3 t^{\prime} t^{\prime}-t^{\prime}-t^{\prime}$, & $t^{\prime} t^{\prime}{ }^{\prime}-t^{\prime}-t^{\prime}+2$ \\
\hline$[7] H R o M s(t)$ & $2 t^{2}$ & $3 t^{2}-2 t$ & $t^{2}-2 t+2$ \\
\hline$H R r T M(n)$ & $n^{2}+4 n+1, n \geq 2$ & $3\left(n^{2}+3 n\right) / 2$ & $\left(n^{2}+n+2\right) / 2$ \\
\hline$K R r T M(n)$ & $\left(5 n^{2}+13 n+2\right) / 2$ & $4 n^{2}+8 n$ & $\left(3 n^{2}+3 n+2\right) / 2$ \\
\hline$H C C(n)$ & $2\left(3 n^{2}+4 n+1\right)$ & $9 n^{2}+9 n+1$ & $3 n^{2}+n+1$ \\
\hline$D D(n)$ & $15 n^{2}+3 n$ & $24 n^{2}$ & $9 n^{2}-3 n+2$ \\
\hline$D D D(n)$ & $15 n^{2}-3 n+6$ & $24 n^{2}-6 n+6$ & $9 n^{2}-3 n+2$ \\
\hline$D_{1}(n)$ & $15 n^{2}-3 n+6$ & $33 n^{2}-19 n+11$ & $18 n^{2}-16 n+7$ \\
\hline$D_{2}(n)$ & $24 n^{2}-16 n+11$ & $42 n^{2}-32 n+16$ & $18 n^{2}-16 n+7$ \\
\hline$D_{3}(n)$ & $33 n^{2}-29 n+16$ & $60 n^{2}-58 n+26$ & $27 n^{2}-29 n+12$ \\
\hline$S D(n)$ & $\left(51 n^{2}-37 n+10\right) / 2$ & $\left(51 n^{2}-61 n+26\right) / 2$ & $51 n^{2}-49 n+16$ \\
\hline
\end{tabular}


International journal on applications of graph theory in wireless ad hoc networks and sensor networks (GRAPH-HOC) Vol.4, No.4, December 2012

TABLE-2

\begin{tabular}{|c|c|c|c|}
\hline Network & Degree & Diameter & Communication Cost \\
\hline [7] HC(n) & 3 & $4 n-1$ & $12 n-3$ \\
\hline [7] HReM(t', $\left.t^{\prime \prime}\right)$ & 3 & $\begin{array}{c}2 t^{\prime \prime}+t^{\prime}-2 \text { for } 2 t^{\prime \prime} \geq t^{\prime} \\
\text { and } 2 t^{\prime}-2 \text { otherwise }\end{array}$ & $\begin{array}{c}\left(6 t^{\prime \prime}+3 t^{\prime}-6\right) \text { for } 2 t^{\prime \prime} \geq t^{\prime} \\
\text { and }\left(6 t^{\prime}-6\right) \text { otherwise }\end{array}$ \\
\hline [7] HRoMs $(t)$ & 3 & $4 t-3$ & $12 t-9$ \\
\hline$H C C(n)$ & 3 & $4 n+3$ & $12 n+9$ \\
\hline$D D(n)$ & 4 & $6 n$ & $24 n$ \\
\hline$D D D(n)$ & 4 & $12 n-6$ & $48 n-24$ \\
\hline$D_{1}(n)$ & 4 & $12 n-6$ & $48 n-24$ \\
\hline$D_{2}(n)$ & 4 & $12 n-6$ & $48 n-24$ \\
\hline$D_{3}(n)$ & 4 & $12 n-6$ & \\
\hline
\end{tabular}

\section{RELATED THEOREMS}

Theorem4.1:[9] A non empty connected graph is Eulerian if and only if it has no vertices of odd degree.

Theorem4.2: [9] A graph is bipartite if and only if it contains no odd cycle.

Theorem4.3: $D D(n), D D D(n)$, and $D_{3}(n)$ are both Euler and bipartite Graphs.

Proof: $D D(n), D D D(n)$, and $D_{3}(n)$ are graphs containing vertices of even degree and does not contain odd cycle, therefore by theorem 4.1 and $4.2, D D(n), D D D(n)$, and $D_{3}(n)$ are Euler graphs and bipartite graphs.

Theorem4.4: $D D(n), D D D(n)$ and $D_{3}(n)$ are bichromatic.

Proof: By theorem $4.3, D D(n), D D D(n), D_{3}(n)$ are bipartite graphs. The vertex set of each graph can be decomposed in to two sets $\mathrm{V}_{1}, \mathrm{~V}_{2}$ such that for any edge $(x, y)$ of $\mathrm{G}, x$ belongs to $\mathrm{V}_{1}$, and $y$ belongs to $\mathrm{V}_{2}$ is always true. Hence $D D(n), D D D(n)$ and $D_{3}(n)$ are bichromatic. $\square$

\subsection{OBSERVATIONS}

4.5.1 $D D(n)$ and $D D D(n)$ can be embedded into Silicate network $S L(n)$ and Dominating Silicate network $\operatorname{DSL}(n)$ respectively with dilation one.

4.5.2 HReM (2n,3n-1), HRoMs $(2 n-1), H C C(n)$, and $\operatorname{HRrTM}(3 n-2)$, can be embedded in to $D_{I}(n)$ with dilation at most 2 , where $n$ is the dimension of $D_{I}(n)$ and $n>1$. 
International journal on applications of graph theory in wireless ad hoc networks and sensor networks (GRAPH-HOC) Vol.4, No.4, December 2012

4.5.3: HReM $(2 n, 3 n-1), \operatorname{HRoMs}(2 n-1), \operatorname{HCC}(n)$ and $\operatorname{HRrTM}(2 n+1)$ can be embedded in to $D_{2}(n)$ with constant dilation 2 , where $n$ is the dimension of $D_{2}(n)$, and $n>1$.

4.5.4: $\operatorname{KRrTM}(3 n-2)$ can be embedded in to $D_{1}(n)$ with dilation one.

4.5.5: $D D(n)$ can be embedded in to $D D D(n)$ with dilation one.

4.5.6: Oxide Network $O X(n)$, Dominating Oxide network $D O X(n)$ can be embedded in to $D D(n)$, $D D D(n)$ respectively with dilation 2.

Oxide network $O X(n)$ and Dominating Oxide network $D O X(n)$ are defined as in $[36,10]$. Now we shall find lower bound for the metric dimension of $D D(n)$.

Theorem4.6: The metric dimension of David Derived network $D D(n)$ is at least $2 n$.

Proof:

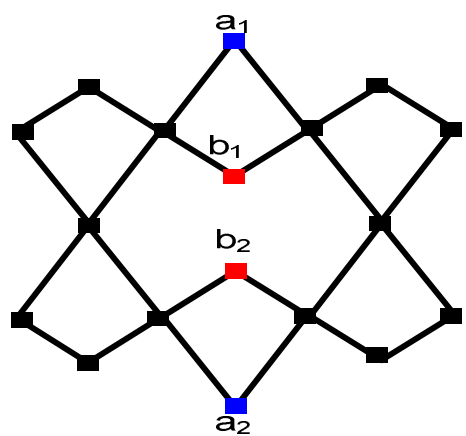

Figure 30(a) $D D(1)$

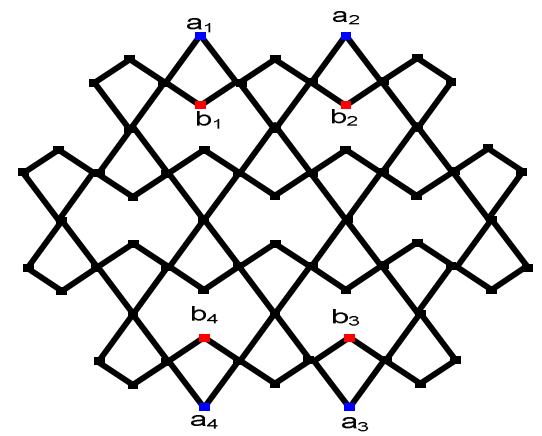

Figure 30 (b) $D D(2)$

For $\mathrm{i}=\mathrm{j}$, each pair of vertices $\left(\mathrm{a}_{\mathrm{i}}, \mathrm{b}_{\mathrm{j}}\right)$ are at equal distance from all other vertices of $\operatorname{DD}(n)$. And there are at least $2 n$ pair of such vertices exist in $D D(n)$, either all $\mathrm{a}_{\mathrm{i}}$ or $\mathrm{b}_{\mathrm{i}}$ must present in the basis. Hence the cardinality of basis must be greater than or equal to $2 n$. Hence the metric dimension of $\mathrm{DD}(n)$ is greater than or equal to $2 n$.

\section{CO ORDINATE SYSTEM FOR $S D(n)$}

A coordinate system is proposed that assigns an address to each vertex of $S D(n)$ as it was proposed for Oxide network in [36], since $S D(n)$ is a proper sub graph of Oxide network $O X(n+1)$ and $O X(n)$ is a proper sub graph of $S D(n+1)$. It is interesting to see that both are identical graph when $n=1$. The basic idea is due to Stojmenovic [7] and to Nocetti et al.[35] who proposed a system for a honeycomb network and a hexagonal network respectively. Three axes, $\alpha, \beta$ and $\gamma$ parallel to three edge directions and at mutual angle of 120 degrees between any two of them are introduced. The three coordinate axes are $\alpha=0, \beta=0$, and $\gamma=0$ respectively. We call lines parallel to the coordinate axes as $\alpha$-lines, $\beta$-lines and $\gamma$-lines.

Here $\alpha=h$ and $\alpha=-k$ are $\alpha$-lines on either side of $\alpha$ - axis. A vertex of $S D(n)$ is assigned a triple $(a, b, c)$ when the vertex is the intersection of lines $\alpha=a, \beta=b$, and $\gamma=c$. See Figure 31 . 
International journal on applications of graph theory in wireless ad hoc networks and sensor networks (GRAPH-HOC) Vol.4, No.4, December 2012

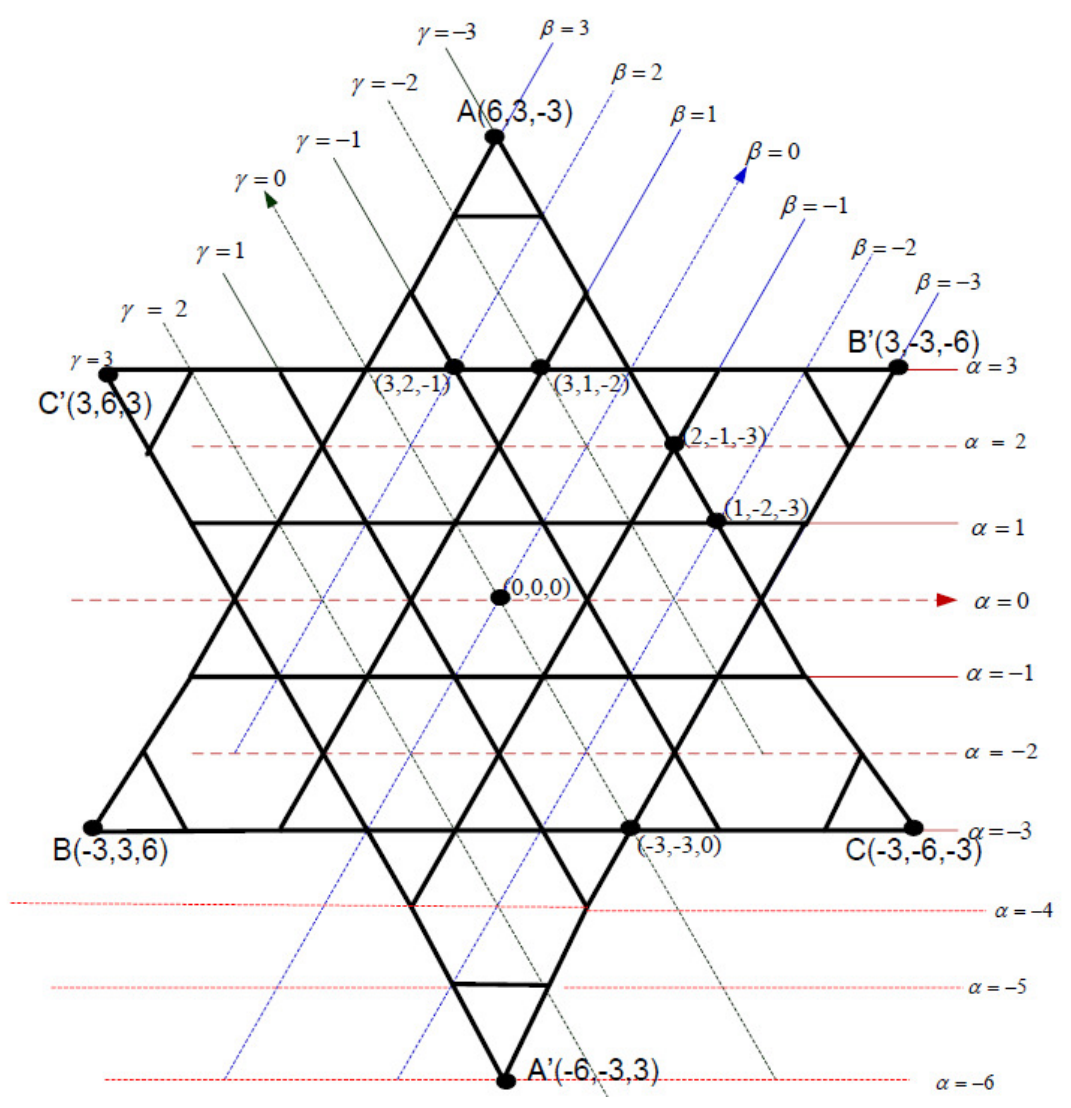

Figure 31. Coordinate System for Star of David Network of dimension 2.

Since $S D(n)$ is symmetrical about $\alpha, \beta$, and $\gamma$ axes , $\mathrm{A}(6,3,-3)$ is the image of $\mathrm{A}^{\prime}(-6,-3,3)$, $\mathrm{B}(-3,3,6)$ is the image $\mathrm{B}^{\prime}(3,-3,-6)$ and $\mathrm{C}(-3,-6,-3)$ is the image of $\mathrm{C}^{\prime}(3,6,3)$.

\subsection{DRAWING ALGORITHM FOR STAR OF DAVID NETWORK OF HIGHER DIMENSION}

Step -1: Draw a Star of David graph $\mathrm{H}$, which is of dimension one (figure 2).

Step-2: Divide each edge into $2^{n}-1$ edges by inserting $2^{n}-2$ vertices at each edge of $H$.

Step-3: Connect all vertices which lies in the same line having odd $\alpha$ values. Repeat this for $\beta$, and $\gamma$ lines also.

Step-4: Insert a new vertex at each new edge crossing.

This will be a Star of David network $S D(n)$ of dimension $n$.

Theorem 5.1: The metric dimension of Star of David network $S D(n)$ is 3 .

Proof: We will prove that $\{\mathrm{A}, \mathrm{B}, \mathrm{C}\}$ is a metric basis for $S D(n)$ (refer Figure 32).

Let $u\left(x_{1}, y_{1}, z_{1}\right)$ and $v\left(x_{2}, y_{2}, z_{2}\right)$ be any two distinct vertices of $G=S D(n)$. Suppose $u$ and $v$ lies in a same $\alpha$ line, then $x_{1}=x_{2}$, and hence either $d(u, \mathrm{~A}) \neq d(v, \mathrm{~A})$ or $d(u, \mathrm{~B}) \neq d(v, \mathrm{~B}) \rightarrow(1)$. 
International journal on applications of graph theory in wireless ad hoc networks and sensor networks (GRAPH-HOC) Vol.4, No.4, December 2012

Similarly, if $u$ and $v$ lies in a same $\beta$ or $\gamma$ lines, then equation (1) is true.

Let $\mathrm{T}_{1}(G)$ be the sub graph of $G$ enclosed by the lines $\alpha=-(2 n-1), \beta=(2 n-1)$ and $\gamma=-(2 n-1)$. And $\mathrm{T}_{2}(\mathrm{G})$ be the sub graph of $\mathrm{G}$ enclosed by the lines $\alpha=(2 n-1), \beta=-(2 n-1)$ and $\gamma=(2 n-1)$.

Clearly $G=\mathrm{T}_{1}(G) \mathrm{U} \mathrm{T}_{2}(G)$ and $\mathrm{T}_{1}(G) \cap \mathrm{T}_{2}(G)$ is a sub graph of Hexagonal network $H X(2 n)$.

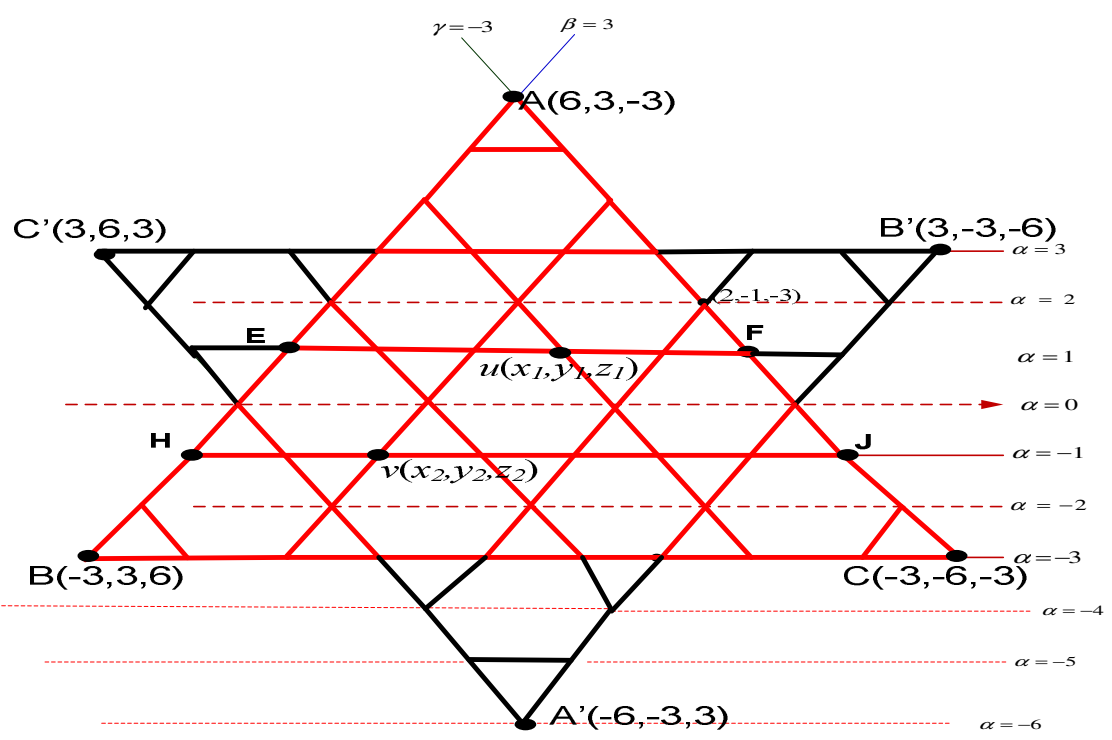

Figure 32 .Edges of equilateral triangle graph $\mathrm{T}_{1}(\mathrm{G})$ is highlighted with red color.

\section{Case1:}

If $u$ and $v$ belongs to $\mathrm{T}_{1}(\mathrm{G})$ and $x_{1}=x_{2}$, then $d(u, \mathrm{~B}) \neq d(v, \mathrm{~B})$ and $d(u, \mathrm{C}) \neq d(v, \mathrm{C}) \rightarrow(2)$

If $u$ and $v$ belongs to $\mathrm{T}_{1}(\mathrm{G})$ and $y_{1}=y_{2}$, then $d(u, \mathrm{~A}) \neq d(v, \mathrm{~A})$ and $d(u, \mathrm{~B}) \neq d(v, \mathrm{~B}) \rightarrow(3)$

If $u$ and $v$ belongs to $\mathrm{T}_{1}(\mathrm{G})$ and $z_{1}=z_{2}$, then $d(u, \mathrm{~A}) \neq d(v, \mathrm{~A})$ and $d(u, \mathrm{C}) \neq d(v, \mathrm{C}) \rightarrow(4)$.

\section{Case 2:}

Similarly the equations (2), (3), and (4) are true in $T_{2}(G)$.

\section{Case 3:}

If $u$ and $v$ belongs to $\mathrm{T}_{1}(\mathrm{G}) \cap \mathrm{T}_{2}(\mathrm{G})$, then the equations (2), (3), and (4) are true.

\section{Case 4:}

If $u$ belongs to $\mathrm{T}_{1}(\mathrm{G})$ and $v$ belongs to $\mathrm{G}-\mathrm{T}_{1}(\mathrm{G})$ then $d(u, \mathrm{~A}) \neq d(v, \mathrm{~A})$ if $x_{1}=x_{2}$, $d(u, \mathrm{~B}) \neq d(v, \mathrm{~B})$ if $\mathrm{y}_{1}=y_{2}$ and $d(u, \mathrm{C}) \neq d(v, \mathrm{C})$ if $\mathrm{z}_{1}=z_{2}$.

\section{Case5:}

$u$ and $v$ are vertices in $\mathrm{T}_{1}(\mathrm{G})$ with $x_{1} \neq x_{2}, \quad y_{1} \neq y_{2}$ and $z_{1} \neq \mathrm{z}_{2}$ if and only if $d(\mathrm{~A}, u) \neq d(\mathrm{~A}, v)$.

Proof: If $u$ and $v$ are vertices in $\mathrm{T}_{1}(\mathrm{G})$ with $x_{1} \neq x_{2}, y_{1} \neq y_{2}$ and $z_{1} \neq \mathrm{z}_{2}$, then there exist two equilateral triangles $\mathrm{t}_{1}$ (AEF) sub graph and $\mathrm{t}_{2}$ (AHJ) sub graph as in figure 31 , if and only if $d(u, \mathrm{~A}) \neq d(v, \mathrm{~A})$.

Similarly we can prove case 6 .

\section{Case 6:}

$u$ and $v$ are vertices in $\mathrm{T}_{2}(\mathrm{G})$ with $x_{1} \neq x_{2}, y_{1} \neq y_{2}$ and $z_{1} \neq \mathrm{z}_{2}$ if and only if $d\left(\mathrm{~A}^{\prime}, u\right) \neq d\left(\mathrm{~A}^{\prime}, v\right)$.

From Case 5 and 6, we get case 7 .

\section{Case7:}

If $u\left(x_{1}, y_{1}, z_{1}\right)$ and $v\left(x_{2}, y_{2}, z_{2}\right)$ are vertices in $S D(n)$ then $d(u, \mathrm{~A})=d(v, \mathrm{~A})$ if and only if 
International journal on applications of graph theory in wireless ad hoc networks and sensor networks (GRAPH-HOC) Vol.4, No.4, December 2012

$d\left(u, \mathrm{~A}^{\prime}\right)=d\left(v, \mathrm{~A}^{\prime}\right)$.

By the above results we get case 8 .

Case8:

If $u\left(x_{1}, y_{1}, z_{1}\right)$ and $v\left(x_{2}, y_{2}, z_{2}\right)$ are vertices in $\mathrm{T}_{2}(\mathrm{G})$ with $x_{1} \neq x_{2}, y_{1} \neq y_{2}$ and $z_{1} \neq \mathrm{z}_{2}$, then implies $d\left(u, \mathrm{~A}^{\prime}\right) \neq d\left(v, \mathrm{~A}^{\prime}\right)$ if and only if $d(u, \mathrm{~A}) \neq d(v, \mathrm{~A})$.

Other possibilities are ruled out by the symmetrical nature of $S D(n)$.

Thus the set $\{\mathrm{A}, \mathrm{B}, \mathrm{C}\}$ is a Metric basis for $S D(n)$.

Hence the Metric dimension of $S D(n)$ is 3 .

Note: $\left\{\mathrm{A}^{\prime}, \mathrm{B}^{\prime}, \mathrm{C}^{\prime}\right\}$ is another Metric basis for $S D(n)$.

\section{CONCLUSION AND FUTURE WORK}

In this paper, four new Interconnection networks, David Derived Network $D D(n)$, Dominating David Derived Network $D D D(n)$, Honeycomb cup Network $H C C(n)$ and Kite Regular Trianguline Mesh $\operatorname{KRrTM}(n)$ were introduced and topological properties were studied. Embedding of poly Honeycomb networks, $H C C(n), K R T M(n)$ in to $D_{2}(n)$ is shown for particular dimensions. Also We have investigated the metric dimension of $S D(n)$ and lower bound of the metric dimension for $D D(n)$. There are many applications of the metric dimension to problems of network discovery and verification [38], pattern recognition, image processing and robot navigation [37], geometrical routing protocols [39], connected joins in graphs[40], and coin weighing problems[41]. The Metric dimension of Oxide, Dominating Oxide network, and Dominating David Derived network are under investigation.

\section{ACKNOWLEDGMENT}

A Special thanks from the Authors to the referees whose vigilant reading has greatly improved the eloquence and conciseness of the presentation.

\section{REFERENCES}

[1] J.M.Xu,"Topological Structure and Analysis of Interconnection Networks", Kluwer Academic Publishers. Norwell 2001.

[2] S. L. Bezrukov, J. D. Chavez, L. H. Harper, M. Röttger and U. P. Schroeder, "Embedding of Hypercubes into Grids," Proceedings of the 23rd International Symposium on Mathematical Foundations of Computer Science, Brno, 24-28 August 1998, pp 693-701.

[3] S. L. Bezrukov, B. Monien, W. Unger and G. Wechsung, "Embedding Ladders and Caterpillars into the Hypercube," Discrete Applied Mathematics, Vol. 83, No. 1-3, 1998, pp 21-29.

[4] P. Manuel, I. Rajasingh, B. Rajan and H. Mercy, "Exact Wire length of Hypercube on a Grid," Discrete Applied Mathematics, Vol. 157, No. 7, 2009, pp1486-1495.

[5] V. Sunitha, "Embedding Some Hierarchical Caterpillars into Hypercube," Electronic Notes in Discrete Mathematics, Vol. 22, No. 10, 2005, pp 387-389.

[6] Narsingh Deo, "Graph theory with applications to Engineering and Computer Science" Prentice -Hall of India private Limited M-97,pp.24-25.

[7] I.Stojmenovic, "Honeycomb Networks:Topological Properties and Communication Algorithms", IEEE Trans.Parallel and Distributed Systems, vol.8, pp1036-1042, 1997.

[8] Star of David [online] available, http://en.wikipedia.org/wiki/Star_of_David.

[9] J . A Bondy and U.S.R Murty "Graph theory with Application" 1976. The Macmillan Press Ltd.

[10] F. Simonraj, A. George "Topological properties few poly Oxide, Poly Silicate , DOX, and DSL Networks", International Journal of Future Computer and Communication ,Vol.2, April 2013, pp 9095.(IACSIT Press). 
International journal on applications of graph theory in wireless ad hoc networks and sensor networks

(GRAPH-HOC) Vol.4, No.4, December 2012

[11] T. Dvôrák, "Dense sets and embedding binary trees into hypercubes", Discrete Applied Mathematics, Vol.155 (4) (2007) pp 506-514.

[12] Y.L. Lai, K. Williams, "A survey of solved problems and applications on bandwidth, edge sum, and profile of graphs", "Journal of Graph Theory", Vol.31 (1999) pp75-94.

[13] J.F. Fang, K.C. Lai,"Embedding the incomplete hypercube in books", Information Sciences Vol.96 (2005) pp 1-6.

[14] P. Manuel, I. Rajasingh, B. Rajan, H. Mercy, "Exact wire length of hypercube on a grid", Discrete Applied Mathematics, Vol.157 (7) (2009) pp1486-1495.

[15] W.K. Chen, M.F.M. Stallmann, "On embedding binary trees into hypercubes”, Journal of Parallel and Distributed Computing, Vol.24 (1995),pp132-138.

[16] J. Fan, X. Jia, "Embedding meshes into crossed cubes", Information Sciences Vol.177 (15) (2007) pp.3151-3160.

[17] S.L. Bezrukov, "Embedding complete trees into the hypercube", Discrete Applied Mathematics Vol.110 (2-3) (2001) pp 101-119.

[18] P.L. Lai, C.H. Tsai, "Embedding of tori and grids into twisted cubes", Theoretical Computer Science Vol.411 (40-42) (2010) pp 3763-3773.

[19] Y. Han, J. Fan, S. Zhang, J. Yang, P. Qian, "Embedding meshes into locally twisted cubes", Information Sciences, Vol. 180 (19) (2010) pp 3794-3805.

[20] X . Yang, Q. Dong, Y.Y. Tan, "Embedding meshes/tori in faulty crossed cubes", Information Processing Letters Vol.110 (14-15) (2010) pp 559-564.

[21] R. Caha, V. Koubek, "Optimal embeddings of generalized ladders into Hypercubes", Discrete Mathematics Vol.233 (2001) pp 65-83.

[22] M. Rottger, U.P. Schroeder, “ Efficient embeddings of grids into grids”, Discrete Applied Mathematics, Vol. 108 (1-2) (2001) pp143-173.

[23] J. Opatrny, D. Sotteau, "Embeddings of complete binary trees into grids and extended grids with total vertex-congestion 1", Discrete Applied Mathematics , Vol.98 (2000) pp 237-254.

[24] J.D. Chavez, R. Trapp, "The cyclic cutwidth of trees”, Discrete Applied Mathematics ,Vol.87 (1998) $5-32$.

[25] C.J. Guu, "The circular wire length problem for hypercubes", $\mathrm{PhD}$ dissertation, University of California, Riverside, 1997.

[26] M.C. Yang, "Path embedding in star graphs", Applied Mathematics and Computation , Vol.207 (2) (2009) pp 283-291.

[27] A. Vodopivec, "On embeddings of snarks in the torus", Discrete Mathematics Vol.308 (10) (2008)1847-1849.

[28] I. Rajasingh, J. Quadras, P. Manuel, A. William, "Embedding of cycles and wheels into rbitrary trees”, Networks Vol.44 (2004) pp 173-178.

[29] I. Rajasingh, B. Rajan, R.S. Rajan, "On embedding of m-sequential k ary trees into hypercubes", Applied Mathematics , Vol.1 (6) (2010) pp 499-503.

[30] C.H. Tsai, "Embedding of meshes in Möbius cubes", Theoretical Computer Science Vol.401 (1-3) (2008) pp181-190.

[31] A.K. Gupta, D. Nelson, H. Wang, "Efficient embeddings of ternary trees into hypercubes", Journal of Parallel and Distributed Computing Vol.63 (6) (2003) pp 619-629.

[32] P. Manuel, "Minimum average congestion of enhanced and augmented hypercube into complete binary tree", Discrete Applied Mathematics Vol.159 (5) (2010) pp 360-366.

[33] P. Manuel, M. Arockiaraj, I. Rajasingh, B. Rajan, "Embeddings of circulant networks", Journal of Combinatorial Optimization, Springer Publication, DOI 10.1007/s10878-011-9443-X.

[34] P. Manuel, M. Arockiaraj, I. Rajasingh, B. Rajan, "Embedding hypercubes into cylinders, snakes and caterpillars for minimizing wire length" , Discrete Applied Mathematics , Vol.159 (17) (2011) pp 2109-2116.

[35] Nocetti F.G, Stojmenovic I, Zhang J, "Addressing and Routing in Hexagonal Networks with Applications for Tracking Mobile Users and Connection Rerouting in Cellular Networks", IEEE Transactions On Parallel and Distributed Systems, vol. 13, pages 963- 971, 2002.

[36] Paul Manuel and Indra Rajasingh, “Topological Properties of Silicate Networks",5th IEEE GCC Conference, Kuwait, March, 16-19, 2009.

[37] S.Khuller, B.Ragavachari, A.Rosenfeld, "Land marks in Graphs", Discrete Applied mathematics, vol 70, pp 217-229.(1996). 
International journal on applications of graph theory in wireless ad hoc networks and sensor networks (GRAPH-HOC) Vol.4, No.4, December 2012

[38] Z.Beerliova, F. Eberhard, T. Erlebach, A. Hall, M.Hoffman, M. Mihal'ak, "Network discovery and Verification," IEEE Journal on selected areas in communications, Vol. 24, no. 12 (2006), pp. 21682181.

[39] K. Liu, N. Abu-Ghazaleh, "Virtual Coordinate Backtracking for Void Traversal in Geographic Routing”, Networking and Internet Architecture,2006.

[40] A. Sebo, E. Tannier , "On Metric Generators of Graphs," Mathematics of Operation research, Vol. 29, no. 2 (2004) pp 383-393.

[41] S.Soderberg, H.S.Shapiro, "A Combinatory Detection problem”, Amer.Math. Monthly , Vol.70 (1963) pp1066-1070.

[42] S.B.Bell , F.C.Holroyd , D.C.Mason , "A Digital Geometry for Hexagonal Pixels," Image and Vision Computing, vol. 7, pp. 194-204, 1989.

[43] B.Rajan , I.Rajasingh, M.C.Monica, P.Manuel . "Metric dimension of enhanced hypercube networks," Journal Combin. Math. Combin. Comput. vol. 67, 5-15, (2008).

[44] B.Rajan, I.Rajan, P.Venugopal , M.C.Monica "Minimum metric dimension of illiac networks," Ars Combin. (Accepted).

[45] M.R.Garey, D.S.Johnson, "Computers and Intractability", A Guide to the Theory of NPCompleteness. Freeman, NewYork (1979).

[46] F.Harary, R.A. Melter " On the metric dimension of a Graph," Ars Combin. vol 2, pp. 191-195 (1976).

[47] M.A.Johnson, "Structure-activity maps for visualizing the graph variables arising in drug design,". Journal of Biopharm. Stat. 3, 203-236 (1993).

[48] S.Khuller, B.Ragavachari., A.Rosenfield "Landmarks in Graphs". Journal of Discret Appl. Math. vol. 70(3), 217-229 (1996).

[49] P.Manuel, M.I.Abd-El-Barr, I.Rajasingh, B.Rajan “An Efficient Representation of Benes Networks and its Applications,"Journal of Discrete Algorithms vol. 6(1), 11-19 (2008).

[50] P.Manuel, B.Rajan , I.Rajasingh, M.C.Monica."On minimum metric dimension of honeycomb networks,” Journal of Discret. Algorithms vol.6(1), pp.20-27 (2008).

[51] P.Manuel, B.Rajan , I.Rajasingh, M.C.Monica. "Landmarks in Torus Networks". Journal of. Discret. Math. Sci.Cryptograph. vol. 9(2), pp.263-271(2006).

[52] P.J.Slater, "Leaves of trees". Congr. Numer. 14, pp.549-559 (1975).

[53] P.J.Slater, "Dominating and reference sets in a graph." , Journal of . Math. Phys. Sci. vol 22(4), pp.445-455 (1988).

[54] G.Chartrand, L.Eroh, M.A.Johnson, O.R. Oellermann, "Resolvability in graphs and the metric dimension of a Graph", Discrete Applied maths.vol .105, pp.99-113 (2000).

[55] B.Rajan, S.K.Thomas, M.C.Monica, "Conditional Resolvability of Honeycomb and Hexagonal Networks," Journal of Mathematics in computer Science. Vol. 5, no.1. (2011), pp 89-99.

\section{AUTHORS}

F.SIMONRAJ received his Master Degree and Master of Philosophy from University of Madras, Chennai, India in 2004 and 2006 respectively. He jointed Hindustan group of Institution in 2005. Now he is doing Doctoral program in Hindustan University, Chennai, India. He is a member of All India Mathematics Teachers Association. He has seven years of teaching experience in Engineering Colleges both undergraduate and post graduate level. His area of interest is Network Designing and Applications of Graph Theory in Ad Hoc Networks and Combinatorial Algorithms.

A.GEORGE received $\mathrm{PhD}$ degree from IIT Madras. He joined in Hindustan Group of Institutions, Chennai in 2001. He is working as a Professor in the department of Information Technology. He has more than 20 years of teaching experience in colleges both undergraduate and post graduate level. He is guiding four research scholars in Hindustan University. He has more than 10 international Journal publications. His research interests are Graph theory applications in Ad Hoc Networks, Fuzzy Theory, and Combinatorial Algorithms.

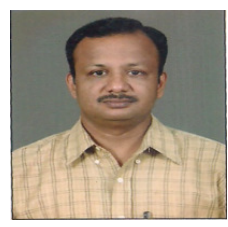

